\title{
Parecoxib: an Enhancer of Radiation Therapy for Colorectal Cancer
}

\author{
Wei Xiong ${ }^{1 \&}$, Wen-Hui Li ${ }^{1 \&}$, Yong-Xin Jiang ${ }^{2 \&}$, Shan Liu ${ }^{2 \&}$, Yi-Qin Ai ${ }^{1}$, Rong Liu ${ }^{3}$, \\ Li Chang ${ }^{1}$, Ming Zhang ${ }^{1}$, Xiao-Li Wang ${ }^{1}$, Han Bai ${ }^{1}$, Hong Wang ${ }^{2}$, Rui Zheng ${ }^{2}$, \\ Jing $\operatorname{Tan}^{1 *}$
}

\begin{abstract}
Background: To study the effect of parecoxib, a novel cyclooxygenase-2 selective inhibitor, on the radiation response of colorectal cancer (CRC) cells and its underlying mechanisms. Materials and Methods: Both in vitro colony formation and apoptosis assays as well as in vivo mouse xenograft experiments were used to explore the radiosensitizing effects of parecoxib in human HCT116 and HT29 CRC cells. Results: Parecoxib sensitized CRC cells to radiation in vitro with a sensitivity enhancement ratio of 1.32 for HCT116 cells and 1.15 for HT29 cells at a surviving fraction of 0.37 . This effect was partially attributable to enhanced apoptosis induction by parecoxib combined with radiation, as illustrated using an in vitro apoptosis assays. Parecoxib augmented the tumor response of HCT116 xenografts to radiation, achieving growth delay more than 20 days and an enhancement factor of 1.53. In accordance with the in vitro results, parecoxib combined with radiation resulted in less proliferation and more apoptosis in tumors than radiation alone. Radiation monotherapy decreased microvessel density (MVD) and microvessel intensity (MVI), but increased the hypoxia level in xenografts. Parecoxib did not affect MVD, but it increased MVI and attenuated hypoxia. Conclusions: Parecoxib can effectively enhance radiation sensitivity in CRC cells through direct effects on tumor cells and indirect effects on tumor vasculature.
\end{abstract}

Keywords: Parecoxib - radiation - colorectal cancer - HCT116 - HT29 - microvessel density - microvessel intensity

Asian Pac J Cancer Prev, 16 (2), 627-633

\section{Introduction}

Preoperative chemoradiotherapy (CRT) is the standard treatment for colorectal cancer (CRC), especially for middle and distal rectal cancers in improving resectability, local control and overall survival (Sauer et al., 2004; Dou et al., 2013). Clinical trials showed that favorable prognoses for patients with CRC who underwent neoadjuvant CRT following radical resection were directly related to the pathological response to CRT (Valentini et al., 2002), especially to the pathological complete response, which was, however, only achieved in 8 29\% of patients (Kim et al., 2006). Improving the effectiveness of radiation on CRC will further improve the outcome of patients.

Cyclooxygenase (COX) enzymes, such as COX-1 and COX-2, are key enzymes for prostaglandin synthesis and are involved in several physiological and pathogenic processes. COX-1 is constitutively expressed in most tissues, playing an important role in the homeostasis of several physiologic functions. In contrast, COX-2 is an inducible enzyme, tightly regulated by various factors including cytokines, growth factors and tumor promoters (Harris et al., 2014). COX-2 may promote the carcinogenesis of many cancers (Tang et al., 2014; Yokouchi et al., 2014). Much attention has been paid to the contribution of COX-2 to early-stage intestinal tumorigenesis. COX-2 inhibitors reduced the risk of developing CRC in patients with familial adenomatous polyposis and in sporadic cases (Dannenberg et al., 2003). COX-2 was overexpressed in CRC cells during carcinogenesis and after radiotherapy and chemotherapy. Increased COX-2 expression was found in tumor cells exposed to radiation and drugs in preclinical experiments and in human CRC tissues (Watwe et al., 2005; Debucquoy et al., 2006; Khan et al., 2011). Chemoradiation-induced COX-2 expression permits cells to resist conventional cancer therapies (Khan et al., 2011). Collectively, these findings suggest that COX-2 may help to radiosensitize CRC cells.

Parecoxib is a novel COX-2 selective inhibitor. Recent studies using xenograft models have demonstrated that parecoxib has greater anti-tumor potency than previous COX-2 inhibitors like celecoxib except for treating pain and inflammation (Senzaki et al., 2008; Kirane et al.,

${ }^{1}$ Department of Radiation Oncology, ${ }^{2}$ Cancer Research Institute of Yunnan Province, Yunnan Cancer Hospital, The Third Affiliated Hospital of Kunming Medical University, ${ }^{3}$ Key Laboratory of Animal Models and Human Disease Mechanisms of Chinese Academy of Sciences \& Yunnan Province, Kunming Institute of Zoology, Kunming, China \&Equal contributors *For correspondence: kmtjing@163.com 
2012), although the mechanism is poorly understood. Furthermore, the combination treatment with parecoxib and other anti-tumor therapies including radiation remains unexplored. Hence, we performed in-vitro colony formation and apoptosis assays and in-vivo mouse xenograft experiments to explore the radiosensitive effect of parecoxib in human HCT116 and HT29 CRC cells.

\section{Materials and Methods}

\section{Cell culture and reagents}

Human HCT116 and HT29 CRC cells were purchased from the cell bank of Kunming Institute of Zoology, Chinese Academy of Sciences (Kunming, China). The cells were cultured in RPMI-1640 medium (Thermo Fisher) supplemented with $10 \%$ fetal bovine serum (Thermo Fisher) and 1\% penicillin/streptomycin (Thermo Fisher) at $37^{\circ} \mathrm{C}$ in a humidified atmosphere with $5 \% \mathrm{CO}_{2}$. The cell lines were confirmed to be pathogen free prior to use. Parecoxib was purchased from Pharmacia and Upjohn Company (Kalamazoo), and $0.9 \%$ saline was used as a vehicle in both in-vitro and in-vivo experiments. As determined previously (data not shown), parecoxib inhibited tumor cell proliferation in vitro in a dosedependent manner, with an $\mathrm{IC}_{50}$ of 390 and $570 \mu \mathrm{mol} / \mathrm{L}$ and an $\mathrm{IC}_{20}$ of 140 and $200 \mu \mathrm{mol} / \mathrm{L}$ for HCT116 and HT29 cells, respectively. Those concentrations of parecoxib were used in the experiments described here.

\section{Western blot analysis}

To determine the constitutive expression of COX-2 and the effects of radiation and parecoxib on COX-2 expression in HCT116 and HT29 cells, we used western blot analysis of protein lysates from cells receiving single or combination treatment at different time points. Cells cultured in six-well plates were exposed to radiation $(0$ or $4 \mathrm{~Gy}$ ) with or without concurrent parecoxib $\left(\mathrm{IC}_{50}\right)$ treatment. The cells were incubated for an additional $24 \mathrm{~h}$ and $72 \mathrm{~h}$ in medium containing either parecoxib or vehicle control. Cell lysates were quantified using BCA Protein Assay Kit (Beyotime). Western blot analysis was performed as described previously with COX-2 (Abcam Ltd.) and $\beta$-actin (Santa Cruz Biotechnology) primary antibodies (Chang et al., 2009).

\section{Colony formation assay}

To determine whether parecoxib affected the radiosensitivity of cancer cells, both HCT116 and HT29 cells were seeded at the density of 200,300,600,2 000, 6 000 or 20000 cells/six-well plate, respectively. $24 \mathrm{~h}$ later, the cells were treated with parecoxib or vehicle control at a concentration equal to the $\mathrm{IC}_{20}$ and immediately irradiated with graded doses $(0,2,4,6,8$, or $10 \mathrm{~Gy})$ of X-rays. The cells were incubated for an additional $48 \mathrm{~h}$ in the presence or absence of parecoxib, and then placed in a parecoxibfree culture medium. 14 days later, the cells were fixed with absolute methanol and stained with $1 \%$ crystal violet (Sigma Chemical Co.). The number of colonies $>50$ cells was counted. Survival fractions (SF) were calculated with the formula: $\mathrm{SF}=$ colonies counted/ (seeded cells $\times$ plating efficiency), where plating efficiency was equal to the mean number of colonies/number of cells inoculated for untreated controls. The SF of cells treated with radiation plus parecoxib were normalized by dividing by the SF of cells treated with parecoxib alone. A survival curve was derived according to the multi-target, single-hit model: $\mathrm{SF}=1-\left(1-\mathrm{e}^{-\mathrm{D} / \mathrm{D} 0}\right)^{\mathrm{N}}$. The sensitivity enhancement ratio (SER) was calculated as the dose of radiation alone divided by the dose of radiation plus parecoxib at a surviving fraction of $37 \%$ (D0).

\section{Flow cytometry analysis of apoptosis}

Cells were seeded into six-well plates at a density of $10^{6}$ cells per well. $24 \mathrm{~h}$ later, the cells were irradiated with various doses of X-rays $(0,4$ or $8 \mathrm{~Gy})$ with or without concurrent parecoxib treatment at a concentration equal to the $\mathrm{IC}_{50}$. The cells were incubated for an additional 48 $\mathrm{h}$ in medium containing either parecoxib or vehicle, and then harvested (retaining all floating cells), fixed in $75 \%$ ethanol at $4^{\circ} \mathrm{C}$ overnight, and incubated with $50 \mu \mathrm{g} / \mathrm{mL}$ propidium iodide (Sigma), 0.3\% NP-40 (Sangon Biotech) and $100 \mu \mathrm{g} / \mathrm{mL}$ RNase A for $30 \mathrm{~min}$ at room temperature (Sangon Biotech). The number of cells that underwent apoptosis (sub-G1) was evaluated using BD Accuri C6 (Becton Dickinson). All experiments were repeated at least three times.

\section{Tumor model and treatment}

Six-week-old male nude mice were obtained from Hunan SJA Laboratory Animal Co. Ltd. (Changsha, China) and housed under standard pathogen-free conditions with 24-h access to food and water. All studies using mice were conducted in accordance with the National Institutes of Health Guidelines for the Care and Use of Laboratory Animals. The Committee on the Use of Live Animals in Teaching and Research, Kunming Medical University (Kunming, China) approved the study protocol.

HCT 116 cells $\left(5 \times 10^{6}\right)$ were injected subcutaneously into the upper left flank region of mice. Treatment started when the diameter of the subcutaneous tumor reached approximately $5 \mathrm{~mm}$. The mice were randomized into 4 groups: control, parecoxib monotherapy, radiation monotherapy and radiation plus parecoxib therapy. When the tumor reached a size of $5 \mathrm{~mm}$, parecoxib was initiated (d0). A parecoxib dose of $30 \mathrm{mg} / \mathrm{kg} / \mathrm{d}$ or vehicle was administered daily by intraperitoneal injection for 14 consecutive days. Radiation was concurrently initiated on $\mathrm{d} 0$ and applied once every other day $\left(\mathrm{d}_{0,2,4,6}\right)$ for a total of 4 treatments. Tumors were irradiated with 6-Mv X-rays at $2 \mathrm{~Gy}$ per fraction using a radiation field of $20 \times 20 \mathrm{~mm}$ covering the entire tumor, with other regions of the mice shielded with lead.

Tumor volume was detected every 5 days and calculated as: $(\mathrm{L} \times \mathrm{W} \times \mathrm{W}) / 2$, where $\mathrm{L}$ and $\mathrm{W}$ respectively represented the longest length and width of the tumor. The mice were sacrificed on $\mathrm{d} 30$ after initiation of treatment regimen, and excised tumors were fixed in paraffin for immunohistochemical analysis and apoptosis assays. To evaluate the vascular condition and blood supply in the xenografts, the microvessel density (MVD), microvessel intensity (MVI) and hypoxia level were detected by their respective markers: CD31, NG2 proteoglycan and 
A

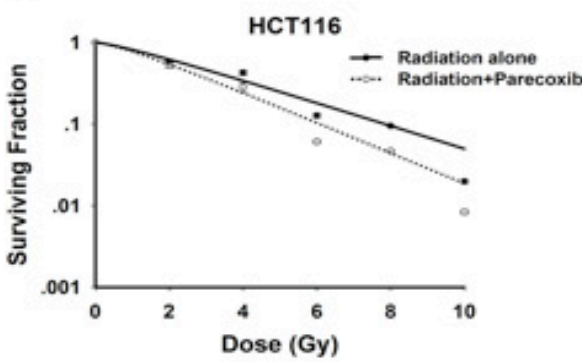

B

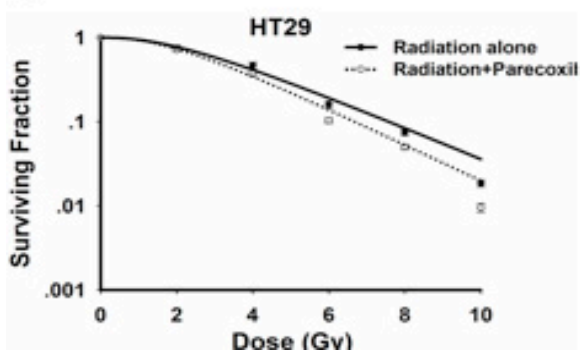

C

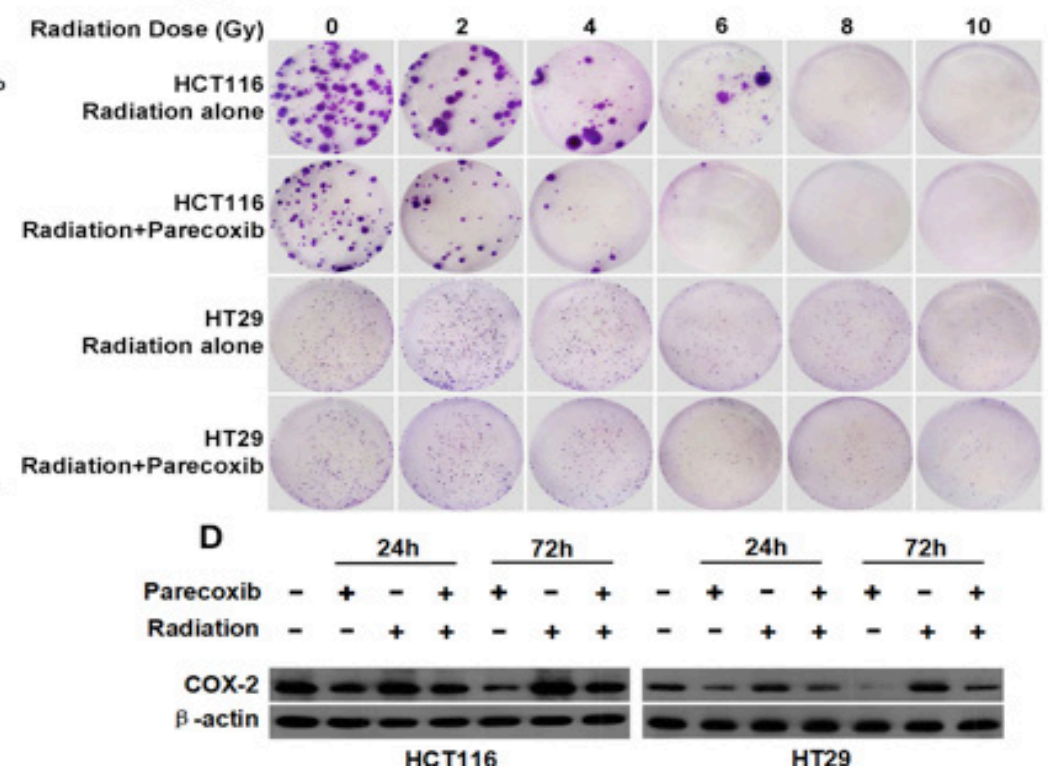

Figure 1. Effect of Parecoxib Combined with Radiation on Survival and COX-2 Expression in CRC Cells. Clonogenic survival curves following radiation with/without parecoxib treatment in HCT116 (A) and HT29 (B) cells. Bars represent standard deviation (SD) of three independent experiments. (C) Representative crystal violet staining of HCT116 and HT29 colonies formed after irradiation with graded doses of X-rays with/without parecoxib treatment. (D) Effect of parecoxib combined with radiation on COX-2 expression in HCT116 and HT29 cells at 24h and 72h detected by western blot.

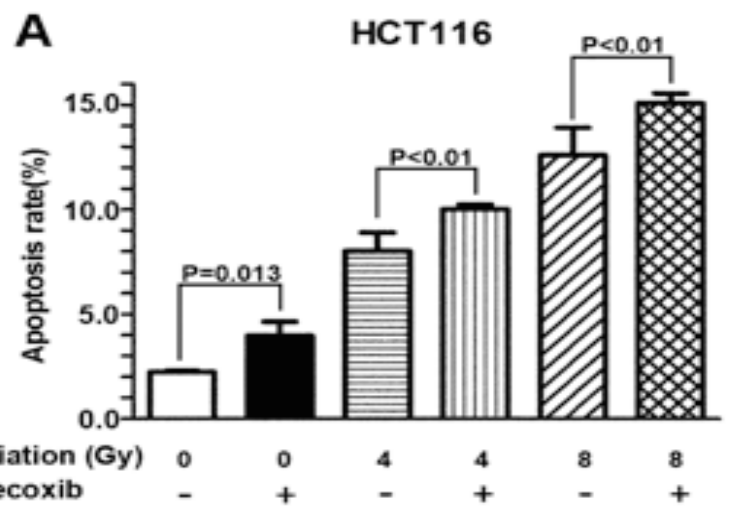

B

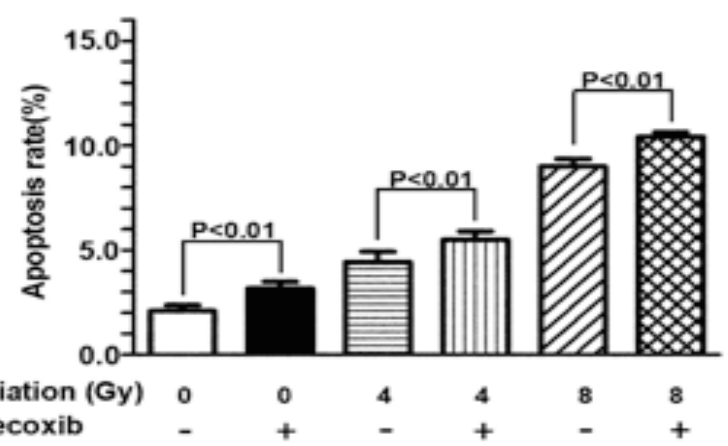

Figure 2. Parecoxib Enhanced Apoptosis in Irradiated HCT116 (A) and HT29 (B) Cells in vitro. Cells were irradiated with X-rays $(0,4$ or $8 \mathrm{~Gy})$ with/without parecoxib treatment for $48 \mathrm{~h}$. Apoptosis was measured using a TUNEL assay. Bars represent $\mathrm{SD}$ of three independent experiments

hypoxia-inducible factor $1 \alpha(\mathrm{HIF}-1 \alpha)$. COX-2 expression was analyzed to evaluate the effects of parecoxib. Growth delay (GD) was calculated as the time for the treated tumors to reach an average volume of $0.8 \mathrm{~cm}^{3}$ minus the time for the control tumors to reach an average volume of $0.8 \mathrm{~cm}^{3}$. Enhancement factor (EF) was calculated as: $\mathrm{EF}=(\mathrm{GD}$ parecoxib plus radiation-GD parecoxib)/GD radiation (Pyo et al., 2001).

\section{Immunohistochemistry and apoptosis analysis of xenografts}

Tumor tissues on $\mathrm{d} 30$ after initiation of treatment were fixed, embedded, and sliced into thick sections for immunohistochemistry. Antibodies for COX-2 (Abcam Ltd.), HIF-1 $\alpha$ (Abcam Ltd.), NG2 (Abcam Ltd.), Ki-67 (Santa Cruz Biotechnology) and CD31 (Abcam Ltd.) were used to detect the respective proteins in the tumors.

To assess apoptosis in the tumor, a terminal transferase dUTP nick end labeling (TUNEL) Assay Kit (KeyGen) was used according to the manufacturer's protocol.
Statistical data analysis

Statistical analysis was performed by one-way analysis of variance (ANOVA). The data were expressed with mean \pm standard deviation $(\mathrm{x} \pm \mathrm{s}) . p<0.05$ was considered to be statistically significant.

\section{Results}

\section{COX-2 expression in HCT116 and HT29 cells}

Untreated HCT116 cells exhibited relatively high constitutive COX-2 expression, whereas the untreated HT29 cells had lower COX-2 expression (Figure 1D). Both cell lines exhibited enhanced COX-2 expression in a time-dependent manner after exposure to radiation. Parecoxib attenuated the radiation-enhanced COX-2 expression at $24 \mathrm{~h}$ and $72 \mathrm{~h}$ after treatment.

Sensitivity of CRC cells to radiation by parecoxib in vitro

The SF of HCT116 and HT29 cells in the colony formation assay declined in a radiation dose-dependent 


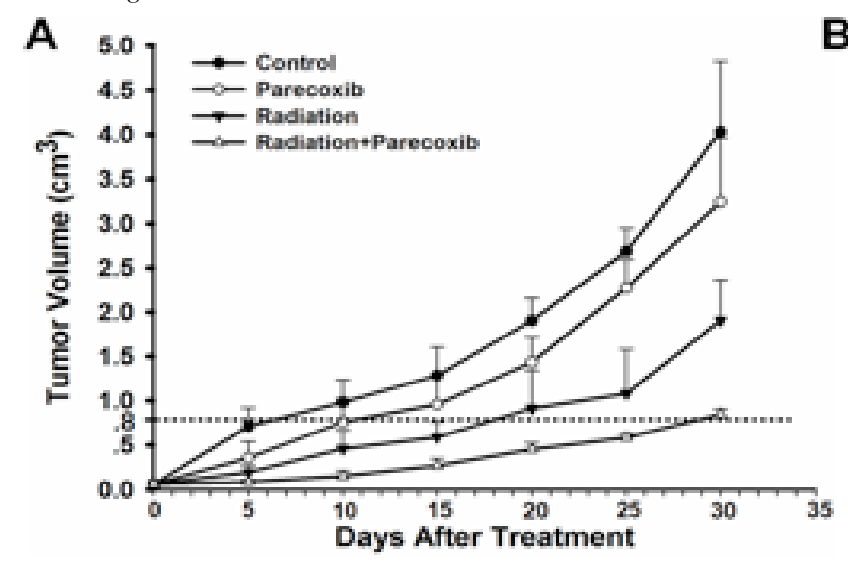

C Tumor Volume of Day 30 After Treatment

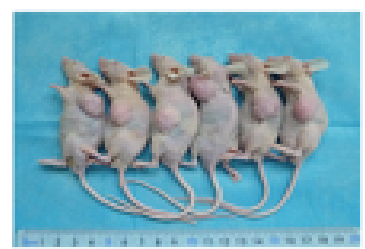

Control

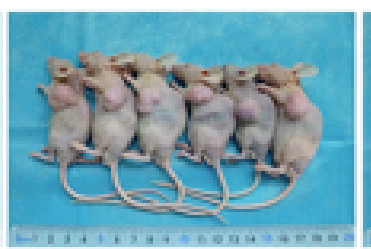

Parecoxib
B

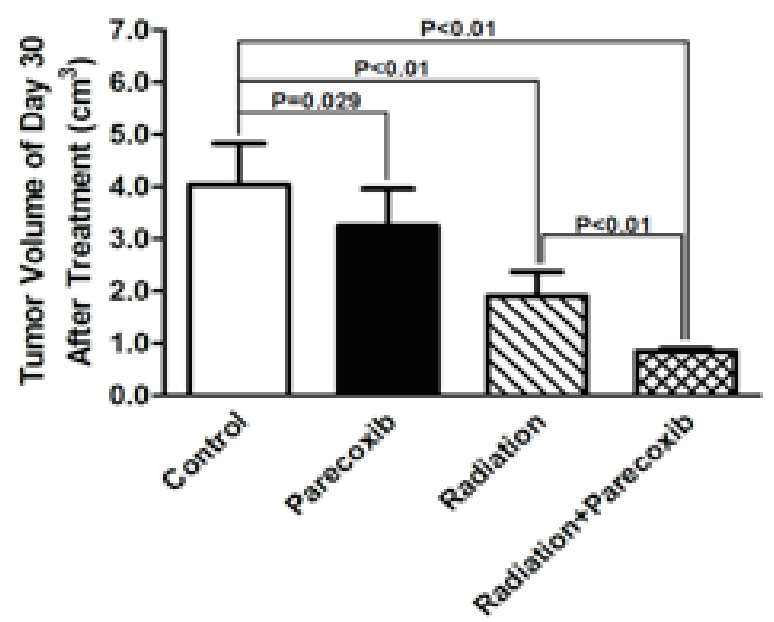

Figure 3. Parecoxib Augmented the in-vivo Tumor Response of CRC Xenografts to Radiation. (A) HCT116 cells were injected subcutaneously into nude mice and treated with parecoxib/vehicle, with/without radiation. Growth delay (GD) was calculated as the time for treated tumors to reach an average volume of $0.8 \mathrm{~cm}^{3}$ minus the time for control tumors to reach $0.8 \mathrm{~cm}^{3}$. $(\mathbf{B}, \mathbf{C})$ Tumor volume in each group on $\mathrm{d} 30$ after treatment initiation

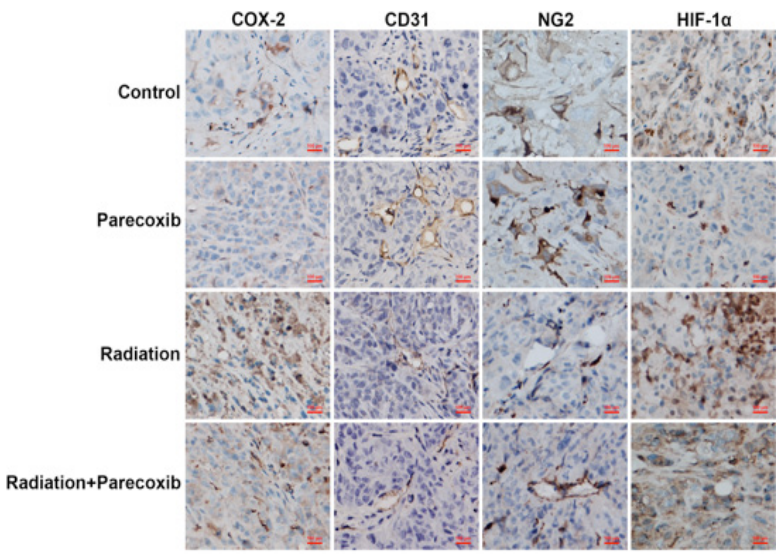

Figure 4. Parecoxib Reversed Radiation-induced Hypoxia and Abnormal Tumor Vasculature. Immunohistochemical staining for COX-2, CD31, NG2 and hypoxia-inducible factor $1 \alpha(\mathrm{HIF}-1 \alpha)$ was performed in tumor tissues collected on $\mathrm{d} 30$ after treatment initiation. Control tumors constitutively expressed COX-2. COX-2 level was significantly elevated in irradiated tumors. Radiation monotherapy decreased CD31 and NG2 proteoglycan expression, but significantly enhanced HIF-1 $\alpha$ expression. Parecoxib inhibited both the constitutive and radiation-induced COX-2 expression but did not affect CD31 level in tumors treated with parecoxib monotherapy or combination treatment, respectively. Compared with the radiation monotherapy group, the combination treatment group showed increased NG2 proteoglycan expression and reduced hypoxia

manner (Figure 1A C). The SER on d0 was 1.32 and 1.15 for HCT116 and HT29 cells, respectively. These data indicated that parecoxib sensitized both cell lines to radiation and had a stronger effect on HCT116 cells.

Enhancement of apoptosis in irradiated tumor cells by parecoxib in vitro

We used flow cytometry to determine the percentage of cells in the subG ${ }_{1}$ phase, which represented the apoptotic fraction. The parecoxib treatment slightly increased the subG $_{1}$ fraction in both cell lines compared with control cells (Figure 2A, B). When combined with radiation of 4 or $8 \mathrm{~Gy}$, parecoxib significantly enhanced the apoptotic induction compared with radiation alone. The proapoptotic effect of combination treatment was more robust in HCT116 cells than in HT29 cells.

Augment of in-vivo tumor response of CRC xenografts to radiation by parecoxib

No significant weight loss was observed in any of the mice bearing HCT116 subcutaneous xenografts. Compared with the control, $30 \mathrm{mg} / \mathrm{kg}$ of parecoxib alone induced a slight GD; it took approximately 5 additional days for the tumors to reach a volume of $0.8 \mathrm{~cm}^{3}$ (Figure $3 \mathrm{~A})$. Radiation ( $2 \mathrm{~Gy} \times 4$ treatments) extended the GD to more than 10 days. Parecoxib combined with radiation resulted in a dramatic GD more than 20 days. EF in the mice receiving the combination treatment was 1.53 , indicating a synergistic effect of combination treatment (Figure 3B, C).

Effect of parecoxib on the radiation-induced hypoxia and vascular abnormalization

COX-2 was constitutively expressed in the tumors in 


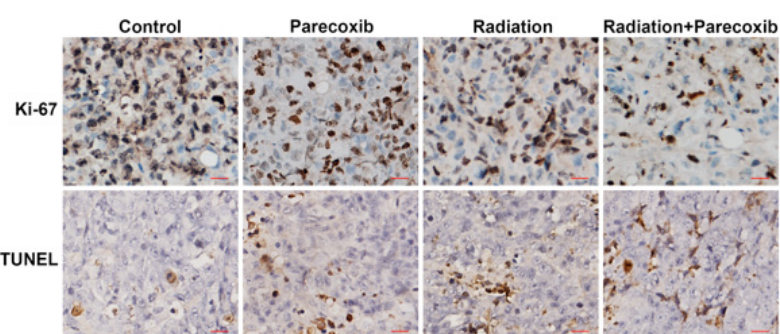

Supplementary Figure 1E. Parecoxib Combined with Radiation Reduced Proliferation and Enhanced

Apoptosis. Immunohistochemical staining for Ki-67 and terminal transferase dUTP nick end labeling (TUNEL) assays were conducted with tumor tissues collected on d30 after initiation of treatment regimen. Compared with control tumors, the parecoxib monotherapy tumors exhibited reduced Ki-67 expression and increased apoptosis. Compared with the radiation monotherapy, the combination treatment decreased cell proliferation, but increased apoptosis

the control mice, and its level was significantly elevated in irradiated tumors (Figure 4). Radiation monotherapy decreased CD31 expression and NG2 proteoglycan level, but significantly enhanced HIF-1 $\alpha$ expression, indicating that there is insufficient blood supply, disorganized vascular structure and enhanced hypoxia level in the irradiated tumors. Parecoxib inhibited the constitutive and radiation-induced $\mathrm{COX}-2$ expression levels in the control and combination-treated tumors, respectively. Compared with the tumors in the radiation monotherapy group, those in the combination therapy group showed markedly-increased NG2 proteoglycan expression and apparently-reduced hypoxia level. However, no significant differences in CD31 expression were observed between control tumors and tumors treated with parecoxib monotherapy or those treated with radiation monotherapy and combination treatment, respectively, indicating that parecoxib did not affect MVD, but did promote vascular normalization via improvement of vascular integrity and reverse of hypoxia status in irradiated tumors.

Effect of parecoxib combined with radiation on the cell proliferation and apoptosis in irradiated tumors

Compared with the control, respective parecoxib and radiation monotherapies reduced Ki-67 expression, but increased apoptosis in the tumors (Supplementary Figure 1E). Parecoxib combined with radiation reduced cell proliferation and enhanced apoptosis to a greater extent than either monotherapy.

\section{Discussion}

Parecoxib is a novel COX-2 selective inhibitor with excellent anti-inflammatory and analgesic effects. In addition, its anti-tumorigenic effects were demonstrated in a preclinical study, in which parecoxib reduced the number of polyps in a mouse model of familial adenomatous carcinoma, and decreased the incidence and number of malignant colorectal tumors by $50 \sim 84 \%$ in azoxymethane-treated rats (Kiguchi et al., 2007). In the present study, we explored the potential of parecoxib to affect the radiosensitivity of CRC cells in vitro and xenograft models.
We used two sorts of CRC cells lines, HCT116 and HT29, which express COX-2 at high and low levels, respectively. The colony formation assay showed that parecoxib effectively enhanced the effects of radiation in both cell lines with a SER of 1.32 and 1.15 for HCT116 and HT29 cells, respectively. The in-vitro radiationenhancing effect of parecoxib seemed to be associated with COX-2 expression level in the cells.

In both cell lines, the $\mathrm{IC}_{50}$ of parecoxib alone induced apoptosis slightly. Parecoxib combined with various doses of radiation produced an additive effect on apoptosis in the HT29 cells compared with each treatment alone, and the effect was even more pronounced in HCT116 cells. Therefore, parecoxib enhanced radiation-induced apoptosis in a COX-2-dependent manner, which was consistent with a recent study (Kim et al., 2013). However, other studies reported contradictory results regarding the relationship between COX-2 expression level and enhancement of radiation effects or radiation-induced apoptosis by selective COX-2 inhibitors (Pyo et al., 2001; Shin et al., 2005). The underlying mechanisms are likely complicated, and further investigations using additional cell lines are required before they can be clearly defined. Regardless, our data show that parecoxib has the radiosensitizing effect on CRC cells and works at least in part via apoptosis induction.

The in-vivo anti-tumor effect of parecoxib monotherapy has been debated. Previous studies reported that parecoxib inhibited tumor growth in a dose-dependent manner in CRC mouse models at doses above $3 \mathrm{mg} / \mathrm{kg} / \mathrm{d}$ (Senzaki et al., 2008; Kirane et al., 2012). In contrast, other studies using a mouse colorectal peritoneal carcinomatosis model and a rat brain tumor model failed to find an anti-tumor effect of parecoxib (Koppe et al., 2006; Eberstål et al., 2012), even at the highest dose of $25 \mathrm{mg} / \mathrm{kg} / \mathrm{d}$. The dosedependent anti-tumor effect of parecoxib observed in previous studies prompted us to use a higher dose (30 $\mathrm{mg} / \mathrm{kg} / \mathrm{d}$ ) in this study, which inhibited the tumor growth effectively on d30 compared with the control. In order to clarify the underlying mechanisms, we examined the events occurring in the tumors after treatment. Enhanced apoptosis and decreased cell proliferation were observed in the parecoxib-treated tumors, similar to observations in gallbladder carcinoma and HT29 CRC models (Kiguchi et al., 2007; Kirane et al., 2012). These observations demonstrate that anti-proliferation and apoptosis induction are involved in the anti-tumor effect of parecoxib in vivo.

We investigated whether parecoxib could enhance the anti-tumor effect of radiation in vivo. Parecoxib combined with radiation produced a dramatically enhanced tumor GD with a significant EF (1.53) compared with radiation alone. Although the data show augmented apoptosis induction and proliferation inhibition in the combination therapy group compared with radiation monotherapy group, the mechanisms responsible for in-vivo radiationenhanced effect of parecoxib are likely to be more complex.

We evaluated hypoxia by measuring HIF- $1 \alpha$ protein, a well-known factor that makes tumor cells resistant to radiotherapy. It was found that the level of hypoxia significantly enhanced in the tumors about 3 weeks after 
the final radiation treatment. It was confirmed a radiationinduced decrease of MVD levels in irradiated tumors at the same time point. Previous studies on murine melanoma and prostate tumors revealed a similar phenomenon, in which reduction of MVD and blood perfusion was observed from one day up to several weeks after radiation (Tsai et al., 2005; Chen et al., 2009). The effect was attributed to early apoptosis in endothelial cells and radiationmediated disruption of angiogenesis several weeks after treatment. In those studies, angiogenesis was defective or ineffective after radiation because the radiation damaged the endothelial cells. Moreover, similar observations were detected by magnetic resonance imaging in patients with CRC who underwent radiotherapy, indicating significant radiation therapy-related reduction in microvascular blood flow in locally advanced CRC (de Lussanet et al., 2005).

Radiation treatment significantly reduced NG2 level, a marker for microvessel pericytes (Raza et al., 2010). Pericytes are important components of vascular walls, regulating the stabilization and function of vasculatures (Birbrair et al., 2015). The reduced pericyte coverage indicated that blood vessels in the irradiated tumors had a disorganized structure and increased permeability. Taken together, the radiation-induced vascular abnormalities included the reduction of MVD and blood perfusion as well as changes in the vascular structure. Importantly, one consequence of increased vascular permeability and concomitant aberrant blood flow is poor delivery of oxygen to the tissues, which is associated with treatment resistance (Armulik et al., 2005). Parecoxib, alone or combined with radiation, did not affect MVD, but it significantly elevated the expression of NG2, improved the maturity and function of microvessels. The vascular normalization effect of parecoxib has been recently confirmed by two studies using the models of CRC and pancreatic cancer (Kirane et al., 2012a; 2012b). It is plausible that vascular normalization induced by parecoxib enhanced oxygen delivery and reduced the regions of chronic hypoxia caused by radiation in vivo, leading to augmented radiosensitivity and apoptosis in the CRC cells. These results, together with those of in-vitro studies, highlight the possibility that the radiosensitizing effect of parecoxib is caused both by direct action on CRC cells and indirect effects on tumor vasculature.

In summary, parecoxib can enhance the efficacy of radiation in CRC cells in vitro and in vivo. Both direct effects on tumor cells and indirect effects through modulation of vasculature likely contribute to its underlying mechanisms. These findings suggest that parecoxib is a promising enhancer of radiation treatment for CRC cells. It still needs further investigation into the precise mechanisms of the radiosensitizing effect of parecoxib and its potential in clinical application.

\section{Acknowledgements}

This work was supported by National Natural Science Foundation of China (No. 81301825), Research Fund for Doctoral Program of Higher Education of China (No. 20135317120004), Joint Funds of Department of Science and Technology of Yunnan Province and Kunming
Medical University (No. 2013FB167) and Key Project of Department of Education of Yunnan Province (No. 2014Z061).

\section{References}

Armulik A, Abramsson A, Betsholtz C (2005). Endothelial/ pericyte interactions. Circ Res, 97, 512-23.

Birbrair A, Zhang T, Wang ZM, et al (2015). Pericytes at the intersection between tissue regeneration and pathology. Clin Sci (Lond), 128, 81-93.

Chang L, Liu YY, Zhu B, et al (2009). High expression of the circadian gene $\mathrm{mPer} 2$ diminishes the radiosensitivity of $\mathrm{NIH}$ 3 T3 cells. Braz J Med Biol Res, 42, 882-91.

Chen FH, Chiang CS, Wang CC, et al (2009). Radiotherapy decreases vascular density and causes hypoxia with macrophage aggregation in TRAMP-C1 prostate tumors. Clin Cancer Res, 15, 1721-9.

Dannenberg AJ, Subbaramaiah K (2003). Targeting cyclooxygenase-2 in human neoplasia: rationale and promise. Cancer Cell, 4, 431-6.

Debucquoy A, Goethals L, Geboes K, et al (2006). Molecular responses of rectal cancer to preoperative chemoradiation. Radiother Oncol, 80, 172-7.

de Lussanet QG, Backes WH, Griffioen AW, et al (2005). Dynamic contrast-enhanced magnetic resonance imaging of radiation therapy-induced microcirculation changesin rectal cancer. Int J Radiat Oncol Biol Phys, 63, 1309-15.

Dou X, Wang RB, Yan HJ, et al (2013). Circulating lymphocytes as predictors of sensitivity to preoperative chemoradiotherapy in rectal cancer cases. Asian Pac J Cancer Prev, 14, 3881-5.

Eberstål S, Badn W, Fritzell S, et al (2012). Inhibition of cyclooxygenase-2 enhances immunotherapy against experimental brain tumors. Cancer Immunol Immunother, 61, 1191-9.

Harris RE, Casto BC, Harris ZM (2014). Cyclooxygenase-2 and the inflammogenesis of breast cancer. World J Clin Oncol, 5, 677-92.

Khan Z, Khan N, Tiwari RP, et al (2011). Biology of Cox-2: an application in cancer therapeutics. Curr Drug Targets, 12, 1082-93.

Kiguchi K, Ruffino L, Kawamoto T, et al (2007). Therapeutic effect of CS-706, a specific cyclooxygenase-2 inhibitor, on gallbladder carcinoma in BK5.ErbB-2 mice. Mol Cancer Ther, 6, 1709-17.

Kim NK, Baik SH, Seong JS, et al (2006). Oncologic outcomes after neoadjuvant chemoradiation followed by curative resection with tumor-specific mesorectal excision for fixed locally advanced rectal cancer: Impact of postirradiated pathologic downstaging on local recurrence and survival. Ann Surg, 244, 1024-30.

Kim YM, Pyo H (2013). Different cell cycle modulation by celecoxib at different concentrations. Cancer Biother Radiopharm, 28, 138-45.

Kirane A, Toombs JE, Larsen JE, et al (2012a). Epithelialmesenchymal transition increases tumor sensitivity to COX2 inhibition by apricoxib. Carcinogenesis, 33, 1639-46.

Kirane A, Toombs JE, Ostapoff K, et al (2012b). Apricoxib, a novel inhibitor of COX-2, markedly improves standard therapy response in molecularly defined models of pancreatic cancer. Clin Cancer Res, 18, 5031-42.

Koppe MJ, Oyen WJ, Bleichrodt RP, et al (2006). Combination therapy using the cyclooxygenase-2 inhibitor Parecoxib and radioimmunotherapy in nude mice with small peritoneal metastases of colonic origin. Cancer Immunol Immunother, $\mathbf{5 5}, 47-55$.

Matsumoto S, Yasui H, Batra S, et al (2009). Simultaneous 
imaging of tumor oxygenation and microvascular permeability using Overhauser enhanced MRI. Proc Natl Acad Sci USA, 106, 17898-903.

Pyo H, Choy H, Amorino GP, et al (2001). A selective cyclooxygenase-2 inhibitor, NS-398, enhances the effect of radiation in vitro and in vivo preferentially on the cells that express cyclooxygenase-2. Clin Cancer Res, 7, 2998-3005.

Raza A, Franklin MJ, Dudek AZ (2010). Pericytes and vessel maturation during tumor angiogenesis and metastasis. Am J Hematol, 85, 593-8.

Sauer R, Becker H, Hohenberger W (2004). Preoperative versus postoperative chemoradiotherapy for rectal cancer. $N \mathrm{Engl}$ J Med, 351, 1731-40.

Senzaki M, Ishida S, Yada A, et al (2008). CS-706, a novel cyclooxygenase-2 selective inhibitor, prolonged the survival of tumor-bearing mice when treated alone or in combination with anti-tumor chemotherapeutic agents. Int $J$ Cancer, 122, 1384-90.

Shin YK, Park JS, Kim HS, et al (2005). Radiosensitivity enhancement by celecoxib, a cyclooxygenase (COX)2 selective inhibitor, via COX-2-dependent cell cycle regulation on human cancer cells expressing differential COX-2 levels. Cancer Res, 65, 9501-9.

Tang SC, Chen YC (2014). Novel therapeutic targets for pancreatic cancer. World J Gastroenterol, 20, 10825-44.

Tsai JH, Makonnen S, Feldman M, et al (2005). Ionizing radiation inhibits tumor neovascularization by inducing ineffective angiogenesis. Cancer Biol Ther, 4, 1395-400.

Yokouchi H,Kanazawa K, Ishida T, et al (2014). Cyclooxygenase-2 inhibitors for non-small-cell lung cancer: A phase II trial and literature review. Mol Clin Oncol, 2, 744-50.

Valentini V, Coco C, Picciocchi A, et al (2002). Does downstaging predict improved outcome after preoperative chemoradiation for extraperitoneal locally advanced rectal cancer? A long-term analysis of 165 patients. Int J Radiat Oncol Biol Phys, 53, 664-74.

Watwe V, Javle M, Lawrence D, et al (2005). Cyclooxygenase-2 (COX-2) levels before and after chemotherapy: a study in rectal cancer. Am J Clin Oncol, 28, 560-4. 